\title{
Integrable Mott insulators driven by finite electric field
}

\author{
Marcin Mierzejewski, ${ }^{1}$ Janez Bonča, ${ }^{2,3}$ and Peter Prelovšek ${ }^{2,3}$ \\ ${ }^{1}$ Institute of Physics, University of Silesia, 40-007 Katowice, Poland \\ ${ }^{2}$ Faculty of Mathematics and Physics, University of Ljubljana, SI-1000 Ljubljana, Slovenia \\ ${ }^{3}$ J. Stefan Institute, SI-1000 Ljubljana, Slovenia
}

\begin{abstract}
We develop a method for extracting the steady nonequilibrium current from studies of driven isolated systems, applying it to the model of one-dimensional Mott insulator at high temperatures. While in the nonintegrable model the nonequilibrium conditions can be accounted by internal heating, the integrability leads to a strongly nonlinear dc response with vanishingly small dc conductivity in the linear-response regime. The finding is consistent with equilibrium results for dc limit of the optical conductivity determined in the presence of a weak and decreasing perturbation.
\end{abstract}

PACS numbers: 72.10.-d,71.27.+a,72.10.Bg

Introduction.- Understanding the differences between integrable and nonintegrable systems has been recognized as a mostly theoretical problem. However, recent advances in the physics of ultracold atoms enable direct experimental verification of the underlying theoretical concepts. The presence of conserved quantities (a hallmark of integrability) restricts thermalization processes [1], hence integrable systems may relax to the generalized Gibbs state 2] instead of the thermal state. Qualitative differences between both system types show up also in their linear-response (LR) to an external field $F$. Although integrability is usually broken by $F$, this breaking is not visible in the LR theory, since LR susceptibility is calculated at $F=0$. The role of integrability at finite driving remains important but largely unexplored field. Here we study insulators driven by electric field $F$ and investigate how the integrability-related properties of the LR regime decay/change with increasing driving.

In the integrable metallic systems there is a dissipationless component of the LR optical conductivity $\sigma(\omega)$ at arbitrary temperature $T$ [3]. The difference between integrable and nonintegrable metals persists also beyond the LR regime but only for restricted time of driving [4]. The response of integrable systems is dominated by the Bloch oscillations which, however, are damped due to field-induced breaking of integrability.

In case of Mott insulators at $T>0$, LR has proven to represent by itself a theoretical challenge not fully understood so far, with a transport fundamentally different from a usual picture of a band insulator at $T>0$. It is plausible that generic nonintegrable models do not show dissipationless transport at $T>0$ as characterized by the charge stiffness $D(T)>0$. This seems to be quite settled also for some prototype one-dimensional integrable models within the insulating regime [5, 6], as e.g. the $t-V$ (or anisotropic Heisenberg) model. However, in the latter case also the regular dynamical conductivity $\sigma(\omega \rightarrow 0)$ vanishes in finite systems at $T>0$ [7] (stimulating the scenario of an 'ideal insulator' [3]), but $\sigma(\omega)$ shows at the same time anomalously large finite-size contribution at $\omega \propto 1 / L$ which at the first sight might be consistent with thermodynamic value $\sigma(0)>0$. It should be noted that $\sigma(0)>0$ may appear as a result of steady-current calculations for an insulator with attached leads [8] as well as of the time-evolution analysis [9]. In view of these open questions [10], it is quite unclear which might be the effect of finite but weak driving $F>0$ as well as of small nonintegrable perturbation.

In this Letter we show that for nonintegrable insulator one can indeed reconcile the response to finite-field driving with the LR result if we take into account the energy increase (the Joule heating) and the existence of (time-dependent) quasi-equilibrium after a short-time transient. More dramatic are results for driven integrable insulators. Since $F \neq 0$ breaks integrability large $F-$ response is quite similar to the nonintegrable case. On the other hand, our results for weak field $F \rightarrow 0$ indicate the vanishing of the steady current response $\sigma(0)=I / F$ reviving the claim of an 'ideal insulator' with $\sigma(0) \rightarrow 0$ at $T>0$. Such scenario seems to follow also from the alternative approach calculating LR $\sigma(0)$ with a weak and decreasing integrability-breaking perturbation.

Model.- A one-dimensional system of interacting spinless fermions with periodic boundary conditions is threaded by time-dependent magnetic flux $\phi(t)$

$$
\begin{aligned}
H= & -t_{h} \sum_{j}\left\{\mathrm{e}^{i \phi(t)} c_{j+1}^{\dagger} c_{j}+\text { h.c. }\right\} \\
& +V \sum_{j} \hat{n_{j}} \hat{n}_{j+1}+W \sum_{j} \hat{n_{j}} \hat{n}_{j+2},
\end{aligned}
$$

where $\hat{n_{j}}=c_{j}^{\dagger} c_{j}, t_{h}$ is the nearest-neighbor (nn) hopping integral, $V$ and $W$ are the nn and next nn repulsive potentials, respectively. In the absence of external driving the system is integrable for $W=0$, whereas nonvanishing $W$ breaks the integrability. As we investigate the physics of Mott insulators, we consider a half-filled system with $L$ sites and $L / 2$ fermions. Initially $\phi(t=0)=0$ and the system is assumed to be in an (approximate) microcanonical state. Then, for $t>0$ the flux $\phi(t)=-F t$ gives rise to a constant electric field $F$. The field will be expressed in units of $\left[t_{h} / e_{0} a\right]$, where $e_{0}$ is the unit charge 

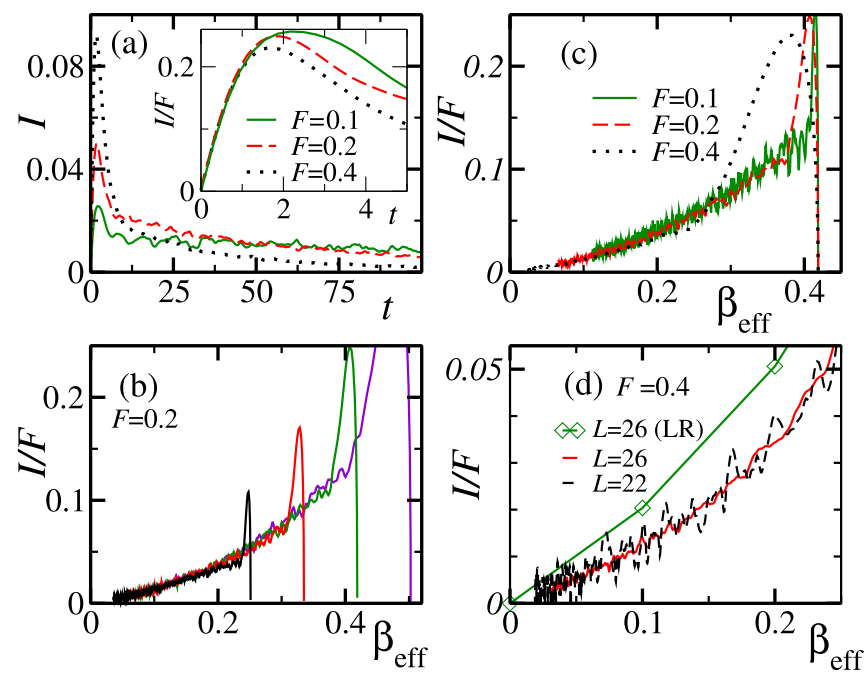

FIG. 1: (Color online) Nonintegrable case $V=3, W=1$, $L=26$ with a pure initial state $\left(N_{s}=1\right)$. (a): current $I(t)$ (main) and $I(t) / F$ (inset) for initial $\beta_{\text {eff }} \simeq 0.42$; (b) and (c): $I / F$ vs. $\beta_{\text {eff }}$ for various initial energies $E_{0}$ (panel b) and various $F$ (panel c); (d): The same as in c) but for various $L$ and compared with the results of equilibrium LR theory.

and $a$ is the lattice distance. We set $t_{h}=\hbar=e_{0}=a=1$. The charge current $I(t)$ can be calculated either directly from the expectation value of the current operator:

$$
\hat{J}=\frac{i}{L} \sum_{j}\left\{\mathrm{e}^{i \phi(t)} c_{j+1}^{\dagger} c_{j}-\text { h.c. }\right\}
$$

or from the increase of the total energy [4]

$$
\dot{E}(t)=\frac{\mathrm{d}}{\mathrm{d} t}\langle H(t)\rangle=L F(t) I(t) .
$$

Calculating the dc current $I$ for finite electric field is a challenging problem. Literally, the dc response (steady nonequilibrium state) is expected only for open quantum systems when the energy gained by carries moving under $F$ [see Eq. (3)] is transferred to the heat reservoirs. In driven isolated systems however, the energy steadily increases causing a steady decay of $I(t)$. This holds true independently of integrability or the system size and remains valid also in the thermodynamic limit. We first analyze nonintegrable systems and show how $I$ can be reliably determined from the decaying $I(t)$.

Nonintegrable case. - As the technical aspects of our computational approach are described in Ref. [4], here we recall only the basic steps. We apply microcanonical Lanczos method [11] and numerically generate $N_{s}$ initial states $\left|\Psi_{l}(0)\right\rangle$ with assumed energy $E_{0}=$ $\langle H(t=0)\rangle$ but as well with a small energy uncertainty $\delta E=\left\langle\left[H(0)-E_{0}\right]^{2}\right\rangle^{1 / 2} \sim 10^{-2}$. Then, the time evolution of $\left|\Psi_{l}(t)\right\rangle$ is calculated by step-vise change of the flux $\phi(t)$ in small time increments $\delta t \ll 1$ applying the Lanczos time propagation method [12] to each interval $\delta t$. The larger the system is, the fewer initial states are needed. It will be shown that for large $L$ one can take $N_{s}=1$ and omit the averaging over $\left|\Psi_{l}(0)\right\rangle$. Similar observation has been reported in Ref. [13] but for relaxation instead of evolution upon driving.

Inset in Fig. 17 shows the ratio $I(t) / F$ for a nonintegrable system. After a relatively short time of driving $(t \sim 2)$ results for different fields split marking the end of the equilibrium LR regime. This nonlinearity becomes even more important for longer driving, when the largest currents occur for the weakest fields (see main panel). In the following we show that such behavior occurs simply due to heating. For this sake it is convenient to introduce a renormalized energy $\varepsilon(t)=\left[E_{\infty}-E(t)\right] / L$, where

$$
E_{\infty}=L(V+W) \frac{L / 2-1}{2(L-1)},
$$

denotes the energy for $T \rightarrow \infty$. In equilibrium and at high temperatures $\varepsilon$ is proportional to the inverse temperature $\beta$

$$
\frac{\varepsilon}{\beta}=\frac{1}{2}+\frac{V^{2}+W^{2}}{16}
$$

We use an effective inverse temperature $\beta_{\text {eff }}$ defined by Eq. (5) also for driven systems, when this quantity should be understood only as a measure of the instantaneous energy. Upon driving $\beta_{\text {eff }}(t)$ monotonically decreases provided $I(t)$ does not change sign.

Fig. 15 shows the same data as panel (a) but the ratio $I / F$ is now plotted as a function of $\beta_{\text {eff }}(t)$ instead of $t$. After the system passes the transient state with a characteristic peak in $I(t)$, results for different $F$ nearly overlap. Hence, the proportionality between $I$ and $F$ is restored far beyond the LR regime provided that the effect of the Joule heating is appropriately subtracted. It also explains the origin of nonlinearity visible in Fig. 1 $\mathrm{h}$ : fixing the time of driving while allowing for different $F$ one actually compares systems with exceedingly different energies. Such inconsistency is not a finite-size effect but is generic for any isolated system with a non-vanishing carrier density. As shown in Fig. \$b, $I\left(\beta_{e f f}\right) / F$ is almost independent of the initial energy $E_{0}$ or the choice of $\left|\Psi_{l}(0)\right\rangle$. This ratio is uniquely determined by the instantaneous energy $\left(\beta_{\text {eff }}\right)$ up to fluctuations which vanish in the thermodynamic limit (see panel d). Fig. 1 also demonstrates that $I\left[\beta_{e f f}(t)\right]$ is close to the the dc current obtained from the equilibrium LR as described in more detail later.

Result presented in Fig. 1 1 consistently support the following picture: the isolated system evolves upon finite $F$ towards the infinite-temperature state but the (quasi) dc current is uniquely determined by the instantaneous energy, suggesting a quasi-equilibrium evolution. Attaching the system to a heat reservoir should set $\beta_{\text {eff }}$ leading to $I\left[\beta_{\text {eff }}(t)\right]=$ const. However, in such case $I$ could depend on the details of the coupling to reservoir. This 

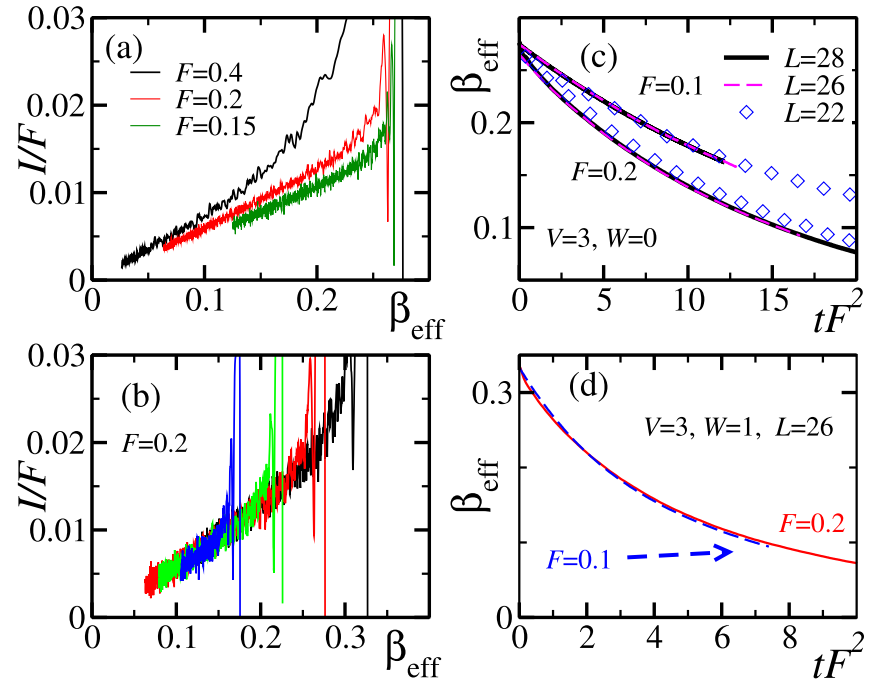

FIG. 2: (Color online) (a) and (b): $I / F$ vs. $\beta_{\text {eff }}$ for integrable system $V=3, W=0$ and $L=28$. (c) and (d) $\beta_{\text {eff }}$ vs. $t F^{2}$ for integrable (c) and nonintegrable (d) systems. In (c) curves are shifted horizontally so they overlap for large times. $N_{s}=1$ apart from $F=0.15$ and $F=0.2$ in (a) where $N_{s}=7$.

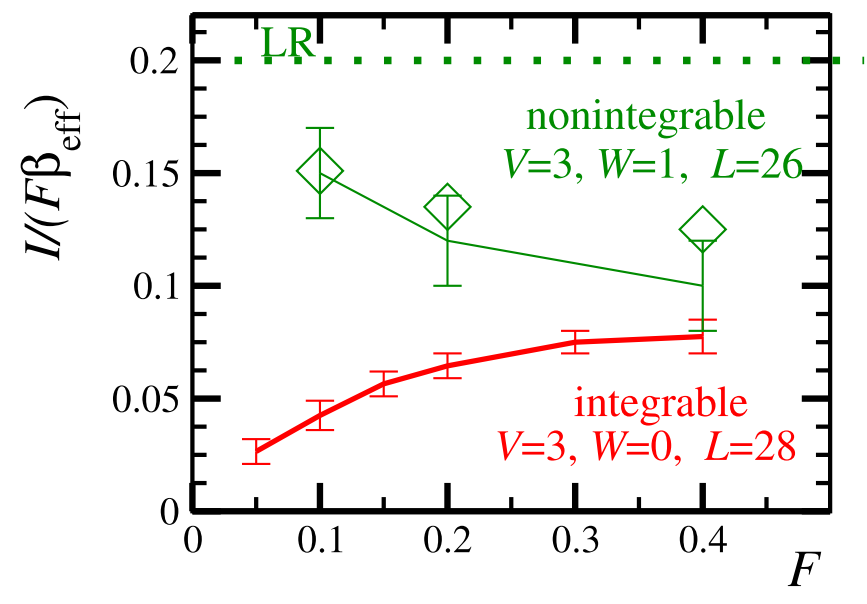

FIG. 3: (Color online) $I /\left(\beta_{e f f} F\right)$ estimated from $E(t)$ [Eq. (3)] for $\beta_{\text {eff }} \rightarrow 0$ (points with guidelines). For nonintegrable case results for $\beta_{\text {eff }}=0.1$ (diamonds) together with LR for $\beta=0.1$ (dotted line) are shown as well.

observation allows us to estimate intrinsic $I$ for finite $F$ even though calculations are carried out on an isolated system. As expected for nonintegrable insulators, the Joule heating represents the central source of nonlinearity at high temperatures.

Integrable case. - Before applying the same approach to the integrable insulators one should address the question whether $I$ can be again uniquely determined (up to finite-size fluctuations) by the instantaneous energy. Since finite $F$ breaks integrability such scenario is possible and as follows from Fig. 2p it actually takes place. Although, for a pure initial state $\left(N_{s}=1\right)$ the finitesize fluctuations are much larger than in the noninte-
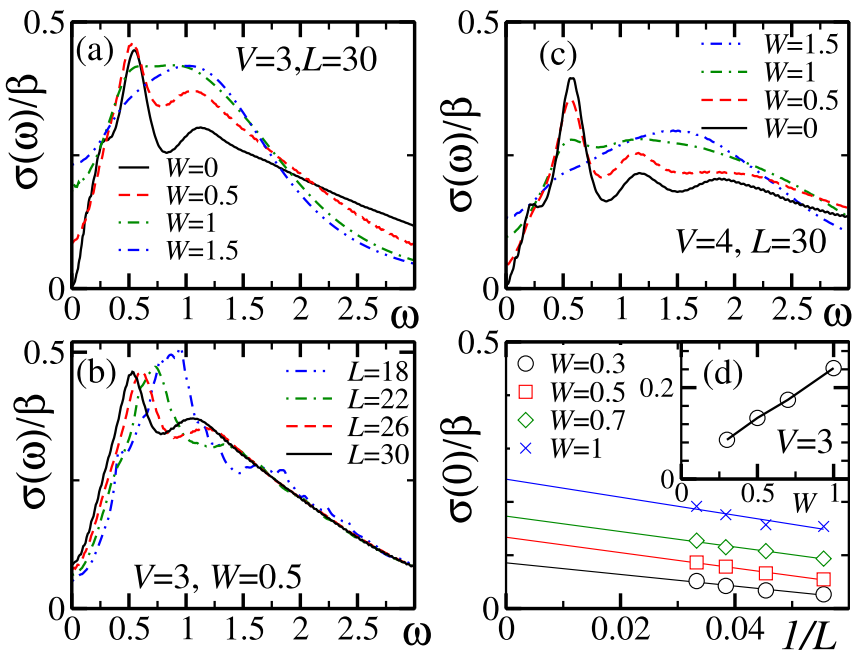

FIG. 4: (Color online) Equilibrium LR: $\sigma(\omega) / \beta$ in the limiting case $\beta \rightarrow 0$ for various $W$ (panels a and c) and various system sizes $L$ (panel b). Main panel in d) shows $\sigma(0) / \beta$ vs. $1 / L$ (points) while inset shows $W$-dependence of $\sigma(0) / \beta$ for $V=3$ extrapolated to $L \rightarrow \infty$.

grable case (Fig. 1k), I( $\left.\beta_{\text {eff }}\right)$ for various initial states do overlap. Therefore, one can apply the same method of reasoning as for nonintegrable insulators and take into account the heating (the energy increase). The results are shown in Fig. 27. Here, one finds important qualitative differences between both system types. While in the nonintegrable case the ratio $I / F$ slightly decreases with $F$ (see Fig. 15), now an opposite dependence is visible. Namely, $I\left(\beta_{\text {eff }}\right) / F$ (strongly) increases with $F$ indicating on a nonlinear mechanism beyond that of the Joule heating. If the dependence between $F$ and $I$ were linear $[I(E, F) / F=\gamma(E)]$, one would obtain from Eq. (3) that $d E / d t \propto F^{2} \gamma(E)$ and all curves in Fig. 2r would overlap. Unlike results for nonintegrable insulators shown in Fig. $2 \mathrm{~d}$, for integrable case the slope of $E(t)$ increases faster than $F^{2}$. Although the accessible values of $L$ do not allow for a detailed size scaling, Fig. 2k strongly suggests that the nonlinearity is not a finite-size effect.

Fig. 3 shows our central result: $F$ dependence of the ratio $I / F$ for $\beta_{\text {eff }} \ll 1$. In the case of nonintegrable insulators, $I / F$ approaches LR $\sigma(0)$ when $F \rightarrow 0$ (dotted horizontal line), while weak but systematic decreasing of this ratio occurs for larger $F$. This is consistent with a nonmonotonic $I-F$ characteristic which has been demonstrated for various tight-binding models [14]. However, in integrable insulator the ratio $I / F$ depends on $F$ in a qualitatively different way and LR regime is not visible down to the lowest accessible fields.

Since the difference due to integrability is most pronounced for low electric fields, the underlying mechanism should be visible also in the LR. Fig. 4 shows equilibrium optical conductivity obtained within the microcanonical Lanczos method introduced in Ref. [7, 11]. As already 
noticed for the integrable insulator $(V>2)[7] \sigma(\omega>0)$ is dominated by a finite-size dependent low frequency peak at $\omega_{0} \sim 16 / L$, its position only weakly varying with $V$. But the existence and the value of $\sigma(0)$ (at any $L$ ) critically depends on $W$ (see Figs. 4a and 4k). Since $W \neq 0$ (as well as $F>0$ ) breaks integrability one can expect finite $\sigma(0)$ in the thermodynamic limit. However the $L \rightarrow \infty$ scaling remains delicate as shown in Fig. 4b for $W=0.5$. For $\omega>\omega_{F S} \simeq 1.5$ all the data form a single $L$-independent curve which could suggest with an oversimplified extrapolation to $L \rightarrow \infty$ that $\sigma(0) / \beta>0.5$. However, the high- $T$ sum rule (at halffilling), $\int_{0}^{\infty} d \omega \sigma(\omega)=\frac{\pi}{4} \beta$, which is moreover $V, W$ independent, requires $L$-independent $\int_{0}^{\omega_{F S}} d \omega \sigma(\omega)$, hence prevents an extrapolation to e.g. $\sigma(0) / \beta>0.4$. A direct scaling of $\sigma(0) / \beta$ suggests $\sigma(0) / \beta \simeq 0.15$ for $W=0.5$ as shown in Fig. 4d. Analogous scaling for other $W>0$ leads to values of $\sigma(0)$ decreasing and (linearly) vanishing with $W$ (see inset in Fig. 4 $\mathrm{d}$ ). In any case, the resulting $\sigma(0)$ is much smaller than straightforward scaling of anomalous fluctuations for $W=0$ which would lead, e.g. for $V=3$, to $\sigma(0)>0.3$ [7]. Clearly, the surprising result $\sigma(0)_{(W \rightarrow 0)} \rightarrow 0$ is consistent with the field dependence $I / F \rightarrow 0$ for $F \rightarrow 0$ shown in Fig. 3. In like manner as $W$, finite $F$ as well breaks integrability, leading effectively to an enhancement of the response function. Consequently $I$ increases with $F$ faster than linearly.

Discussion. - While our numerical results were obtained only for very high $T \gg 0$ it is plausible that their implications might be extended to all $T>0$, especially since at low temperatures strongly nonlinear $I-F$ characteristic is well established [15]. The notion of 'ideal insulator' means vanishing of the $\operatorname{LR} \sigma(0)$ at arbitrary temperature that implies at the same time a nonlinear dc response of integrable insulators down to the lowest electric fields. However, for realistic (open) systems it means that the $\mathrm{LR}$ regime is determined by the mechanisms which break integrability (see discussion in Ref. [6]), hence this regime may terminate at low fields. Here, we present two independent results which support the hypothesis of 'ideal insulator': nonlinear real-time current for finite driving and LR $\sigma(\omega)$ obtained for small but finite integrabilitybreaking parameter $W$. The Anderson insulator (noninteracting case when all states are localized) has been the only well known example of an 'ideal insulator'. An emerging question concerns the differences between the present study and those which suggest $\sigma(0)>0$ [8, 9]. A possible explanation is that in our setup driving doesn't destroy the homogeneous distribution of charge carriers, what may happen, e.g., in an open system driven by a difference in chemical potentials. We have also studied an isolated system exactly at half-filling (microcanonical ensemble). Consequently, the Mott insulating phase is not destroyed (even locally) by a departure from half- filling and integrability is not broken by external leads.

Our results confirm that the Mott insulators, in particular the integrable ones, behave under driving fields qualitatively different from usual band insulators. While in the latter case thermally (as well as photo etc.) induced carriers, i.e. electrons and holes, are quite independent but oppositely charged quasipartilces, in Mott insulators the corresponding doublons and holons are highly correlated, and moreover cannot pass each other in the integrable 1D system (evident in the limit $V>>2$ ). Hence no dc current can be induced at weak $F$ at any $T$. On the one hand, anomalous low-frequency peak at $\omega \propto 1 / L$ clearly requires caution in the interpretation. On the other hand, the concept of the mean free path $\lambda$ would be ill defined if the $1 / L$ dependence in dc response persists also for $L \gg \lambda$.

This work has been supported by the Program P1-0044 of the Slovenian Research Agency (ARRS) and RTNLOTHERM project. M.M. acknowledges support from the N N202052940 project of MNiSW.

[1] M. Kollar, F. A. Wolf, and M. Eckstein, arXiv:1102.2117 P. Barmettler et al, New J. Phys. 12, 055017 (2010).

[2] M. Rigol et al., Phys. Rev. Lett. 98, 050405 (2007); A.C. Cassidy, C.W. Clark, and M. Rigol, Phys. Rev. Lett. 106, 140405 (2011).

[3] X. Zotos and P. Prelovšek, Phys. Rev. B 53, 983 (1996).

[4] M. Mierzejewski and P. Prelovšek, Phys. Rev. Lett. 105, 186405 (2010).

[5] X. Zotos, Phys. Rev. Lett. 82, 1764 (1999); for an overview see F. Heidrich-Meisner, A. Honecker, and W. Brenig, Eur. Phys. J. Special Topics 151, 135 (2007).

[6] J. Sirker, R. G. Pereira, and I. Affleck, Phys. Rev. Lett. 103, 216602 (2009); Phys. Rev. B 83, 035115 (2011).

[7] P. Prelovšek et al, Phys. Rev. B 70, 205129 (2004).

[8] T. Prosen and M. Žnidarič, J. Stat. Mech.: Theory Exp. (2009), P02035; M. Žnidarič, Phys. Rev. Lett. 106, 220601 (2011).

[9] R. Steinigeweg and J. Gemmer, Phys. Rev. B 80, 184402 (2009); R. Steinigeweg, and R. Schnalle, Phys. Rev. E 82, 040103(R) (2010).

[10] C. Buragohain and S. Sachdev, Phys. Rev. B 59, 9285 (1999).

[11] M. W. Long et al, Phys. Rev. B 68, 235106 (2003).

[12] T. J. Park and J. C. Light, J. Chem. Phys. 85, 5870 (1986).

[13] M. Rigol, V. Dunjko, and M. Olshanii, Nature 452, 854 (2008).

[14] M. Mierzejewski et al, Phys. Rev. Lett. 106, 196401 (2011); L. Vidmar et al, Phys. Rev. B 83, 134301 (2011); C. Aron, G. Kotliar, and C. Weber, arXiv: 1105.5387.

[15] T. Oka and H. Aoki, Phys. Rev. Lett. 95, 137601 (2005); A. Takahashi, H. Itoh, and M. Aihara, Phys. Rev. B 77, 205105 (2008); N. Sugimoto, et al, Phys. Rev. B 78, 155104 (2008); M. Eckstein, T. Oka, and P. Werner, Phys. Rev. Lett. 105, 146404 (2010). 